\title{
Corrigendum \\ Focal Electrically Administered Seizure Therapy: A Novel Form of ECT Illustrates the Roles of Current Directionality, Polarity, and Electrode Configuration in Seizure Induction
}

\author{
Timothy Spellman, Angel V Peterchev and Sarah H Lisanby
}

Neuropsychopharmacology (2012) 37, 1077; doi: 10.1038/npp.2011.273

Correction to: Neuropsychopharmacology (2009) 34, 2002-2010; doi:10.1038/npp.2009.12; published online 18 February 2009

We would like to make the following clarifications and corrections to our paper:

In the introduction, we referenced Libet et al (1964) to have found that direct stimulation of motor and somatosensory cortex has revealed lower thresholds with anodal than with cathodal stimulation. In fact, those authors reported the opposite - lower thresholds with cathodal than with anodal stimulation. This result contrasts with the referenced transcranial electrical stimulation studies (Marsden et al, 1982; Rothwell et al, 1987) that found lower threshold with anodal stimulation. The difference in the effect of stimulation polarity in direct cortical stimulation versus transcranial electrical stimulation is not surprising, as the neural activation threshold depends not only on the electrode polarity but also on the electric field distribution relative to the geometry of the targeted neural population, which differs between the two stimulation modalities.

In the reported ECS experiments and in Figures 1 and 4, the negative terminal was defined as the 'anode' and the positive terminal was defined as the 'cathode.' However, in the field of neurostimulation, it is conventional to define the 'anode' as positive and the 'cathode' as negative. This discrepancy stems from the labeling on the experimental ECS device electrodes used in the study, which were labeled 'anode' and 'cathode' to denote electrical current entering and exiting the current source, respectively, following the convention used in some fields of science such as electrochemistry. In contrast, by the neurostimulation convention, the anode and cathode denote current entering and exiting the load connected to the device, respectively.

The EEG sampling frequency was reported to be $100 \mathrm{~Hz}$, whereas the actual sampling frequency was $140 \mathrm{~Hz}$. Furthermore, the EEG data were analyzed with the assumption that the EEG sampling frequency was $128 \mathrm{~Hz}$. Therefore, there is a $-12 \mathrm{~Hz}$ error between the actual sampling frequency and the sampling frequency assumed in the data processing. Consequently, the reported results correspond to EEG band definitions shifted up by $9.4 \%$ : $\delta(1.5-3.8 \mathrm{~Hz}), \gamma(3.8-8.2 \mathrm{~Hz}), \alpha(8.2-13.7 \mathrm{~Hz})$, and $\beta(13.7-$ $32.3 \mathrm{~Hz})$.

These clarifications and corrections do not change the fundamental conclusions of our paper, except for reconsidering the electrode polarity in our finding of polarity dependence of the EEG response to FEAST. Accounting for the electrode relabeling, we found higher ictal power and more lateralization when the anode (positive electrode) is the small anterior electrode and more post-ictal suppression when the cathode (negative electrode) is the small anterior electrode. If we extrapolate from tDCS studies (Lang et al, 2003; Nitsche et al, 2005) that anodal suprathreshold stimulus trains are excitatory, whereas cathodal trains are inhibitory, the increased ictal power and lateralization in the anterior anode FEAST condition may reflect the excitatory effect of anodal stimulation under the anteriorally placed EEG recording leads, whereas the greater postictal suppression seen in anterior cathode FEAST could derive from the inhibitory action of cathodal stimulation.

\section{REFERENCES}

Lang N, Nitsche MA, Paulus W, Rothwell JC, Lemon RN (2003). Effects of transcranial direct current stimulation over human motor cortex on corticospinal and transcallosal excitability. Exp Brain Res 156: 439-443.

Libet B, Alberts WW, Wright Jr EW, DeLattre LD, Levin G, Feinstein B (1964). Production of threshold levels of conscious sensation by electrical stimulation of human somatosensory cortex. J Neurophysiol 27: 546-578.

Marsden CD, Merton PA, Morton HB (1982). Direct stimulation of corticospinal pathways through the intact scalp in human subjects. In: Desmedt J (ed). Motor Control Mechanisms in Health and Disease. Raven Press: New York, NY. pp 387-392.

Nitsche MA, Seeber A, Frommann K, Klein CC, Rochford C, Nitsche MS et al. (2005). Modulating parameters of excitability during and after transcranial direct current stimulation of the human motor cortex. J Physiol 568(Pt 1): 291-303.

Rothwell JC, Thompson PD, Day BL, Dick JPR, Kachi T, Cowan JMA et al. (1987). Motor cortex stimulation in intact man. General characteristics of EMG responses in different muscles. Brain 110: 1173-1190. 